\title{
The effects of enhancing the crosslinking degree in high internal phase emulsion templated poly(dicyclopentadiene) cured by Ring-opening Metathesis Polymerization by a crosslinking comonomer.
}

Efthymia Vakalopoulou and Christian Slugove*

\author{
E. Vakalopoulou, Prof. C. Slugove \\ Institute for Chemistry and Technology of Materials, Graz University of Technology, \\ Stremayrgasse 9, A 8010 Graz, Austria \\ E-mail: slugovc@tugraz.at
}

The effect of enhancing the crosslinking degree in polyHIPEs made from dicyclopentadiene by additionally using a crosslinking comonomer is described. Foams of $80 \%$ porosity with $10-40$ w\% comonomer content in the continues phase are prepared and show similar porosities and morphological characteristics as foams prepared with dicyclopentadiene alone. Assessing the mechanical properties reveals that the ductility is decreasing while the stiffness of the samples is increasing with increasing comonomer content. The foams containing the crosslinking comonomer take up at least their five-fold mass of toluene thereby swelling to at least $30 \mathrm{v} \%$. Upon drying of the swollen specimens, their initial shape and porosity are recovered. This feature distinguishes them from polyHIPEs made from dicyclopentadiene only.

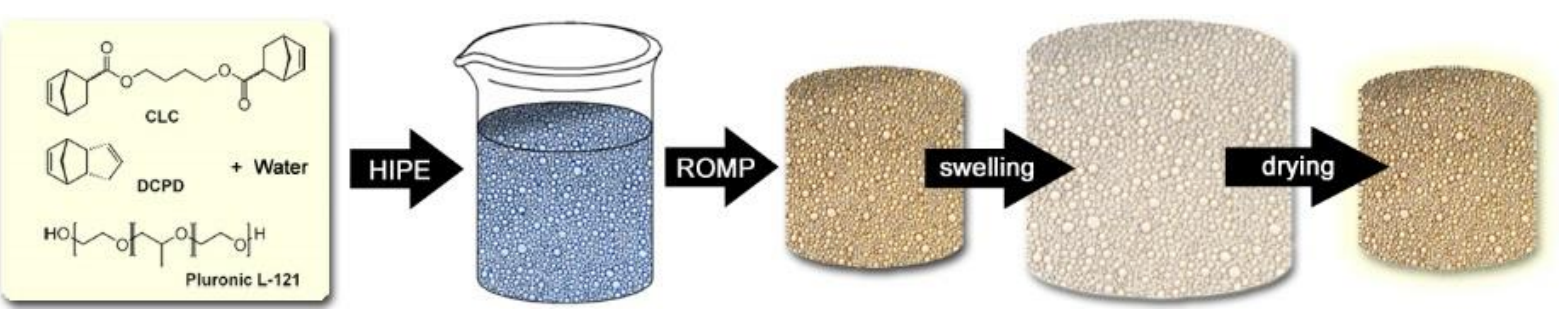




\section{Introduction}

The curing of high internal phase emulsions (HIPE) featuring monomers in its continuous phase is an important and popular way to prepare macroporous polymer foams. ${ }^{[1,2]}$ Such porous materials are often named polymerized HIPEs, abbreviated as poly(HIPE)s. Typically, materials with open-porous highly interconnected architectures are obtained, which find applications in many different fields. ${ }^{[1,2]}$ Very recent examples of their use in separation,,$^{[3,4,5,6]}$ flow-through techniques, ${ }^{[7]}$ biotechnology, ${ }^{[8]}$ drug release,${ }^{[9,10]}$ energy storage,${ }^{[11]}$ sensing ${ }^{[12]}$ or food preservation ${ }^{[13,14]}$ illustrate their versatility. polyHIPEs are prepared by many polymerization techniques but above all (free) radical polymerization of styrenes and electron deficient olefins is used. ${ }^{[1]}$ One of the more exotic polymerization techniques for curing HIPEs, first introduced by Deleuze et al. ${ }^{[15,16]}$ is Ring-opening Metathesis Polymerization (ROMP). ROMP became an important technique, because it enables the preparation of polyHIPEs from dicyclopentadiene (DCPD) as the monomer and the according foams exhibit unique and favorable mechanical properties usually not associated with this class of porous materials. pDCPD based foams are characterized through high strength combined with high toughness. ${ }^{[17,18,19,20]}$ In other words, the porous materials are stiff and ductile at the same time (the Young's modulus of a foam with $80 \%$ porosity is about $116 \mathrm{MPa}$, the modulus of toughness is around $\left.600 \mathrm{~kJ} / \mathrm{m}^{3}\right) .{ }^{[21]}$ Moreover, the high unsaturation of the polymer scaffold allows for a huge variety of chemistries for further functionalizing pDCPD foams. ${ }^{[17,22,23,24,25,26]}$ The combination of these two features led to the use of emulsion templated pDCPD foams as separators in Li-ion batteries, ${ }^{[27]}$ as templates for making porous oxides, ${ }^{[28,29]}$ for making composite materials $^{[28,29,30,31,32,33]}$ and carbon foams, ${ }^{[34]}$ for stabilization of enzymes ${ }^{[35]}$ or for detoxification of nerve gases. ${ }^{[24,25]}$ The most important drawback of pDCPD based polyHIPEs is their relatively fast oxidation in air, ${ }^{[17,24,25]}$ but also the change of the macroscopic shape and porous structure upon drying off of nonpolar solvents is unfavorable. ${ }^{[36]}$ In fact, a shape 
recovery upon drying is worthwhile for all applications which demand a switching between dry and swollen state and for making an a posteriori chemical functionalization of the foams possible.

Herein we report pDCPD based macroporous foams which can be swollen in nonpolar solvents and recover their original shape upon drying. The approach towards the new foams is shown in Figure 1. By using an easily accessible crosslinking comonomer (CLC) bearing two norbornene moiteties in addition to DCPD, the crosslinking degree of the polymer phase is increased and this effect is held responsible for the structure recovery upon drying of swollen monolithic foam specimens. As a side effect, it can be anticipated that free norbornenes further facilitate a postpolymerization functionalization of the foams by e.g. inverse electron demand Diels Alder ${ }^{[23,37,38]}$ or thiol-ene chemistries. ${ }^{[39,40]}$

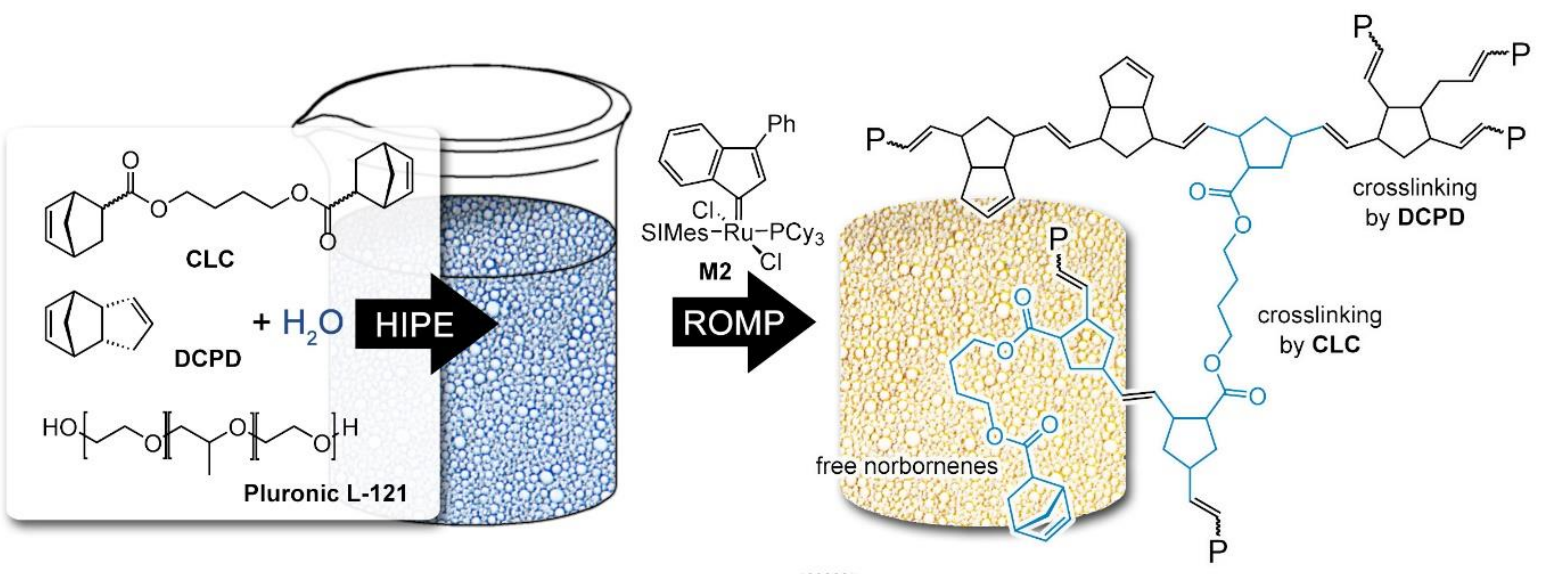

Figure 1. Formulation and process to prepare the foams of this study.

\section{Results and Discussion}

\subsection{Preparation}

The crosslinking comonomer (CLC) was prepared by adding a slight excess of freshly distilled cyclopentadiene to neat butane-1,4-diyl diacrylate under cooling and stirring. Upon 
determining full conversion of the acrylate-groups surplus cyclopentadiene was evaporated under vacuum. Dicyclopentadiene formed as the only byproduct and was removed by flash chromatography. This purification step was done to properly characterize the $\mathbf{C L C}$, however it could be left out in practice because CLC and DCPD are the two monomers employed in the following. Foams of $80 \%$ nominal porosity were prepared with a mixture of DCPD and a varying share of CLC (10-40 wt\% in the mixture with DCPD) as the monomers. Pluronic L121 ( $7 \mathrm{wt} \%$ in respect to the monomer mixture) served as the surfactant for stabilizing the HIPE obtained upon addition of water $(40 \mathrm{~mL})$ to the mixture of the monomers and the surfactant under stirring (Figure 1). The initiator $\mathbf{M} 2^{[41]}(1: 15000$ in respect to the molar amount of norbornene moieties; dissolved in toluene) was used to cure the HIPEs at $80^{\circ} \mathrm{C}$ for $3 \mathrm{~h}$ in closed molds. The resulting polyHIPE specimens were demolded, submersed in acetone and kept there for $15 \mathrm{~min}$. Subsequent drying gave white monolithic macroporous foams. As a reference, samples prepared by using only DCPD as the monomer were prepared similarly. HIPEs containing $50 \mathrm{w} \% \mathbf{C L C}$ or more showed fast phase separation and were not investigated herein.

\subsection{Morphology}

Upon curing the specimens exhibited a volume shrinkage of about $2 \%$ and featured apparent densities in the range from 0.20 to $0.23 \mathrm{~g} \cdot \mathrm{mm}^{-3}$. The skeleton density is about $1.09 \mathrm{~g} \cdot \mathrm{mm}^{-3}$ and is gradually increasing from $\mathbf{P 0}$ to $\mathbf{P 4 0}$. The foams exhibited a porosity close to the nominal porosity of $80 \%$. Measured porosities were in between $79 \%$ and $83 \%$ depending on the method for determining them (Table 1 and vide infra for a discussion). Accordingly, increasing the amount of CLC from 0 to $40 \mathrm{w} \%$ in the formulation did not cause significant changes of densities and porosities of the resulting specimens. In contrast, slight differences in the morphology of the interior of the monoliths could be noted. All specimens exhibited the typical throughout open polyHIPE morphology characterized by the presence of voids, which are 
Table 1. Apparent density, volume shrinkage, porosity and skeleton density of the foams P0P40.

\begin{tabular}{|c|c|c|c|c|c|}
\hline Sample & $\begin{array}{l}\text { Apparent } \\
\text { Density }^{\text {a) }} \\
{\left[\mathrm{g} / \mathrm{cm}^{-3}\right]}\end{array}$ & $\begin{array}{l}\text { Volume } \\
\text { shrinkage b) } \\
{[\%] \mathbf{a}}\end{array}$ & $\begin{array}{l}\text { Porosity } \\
(\text { mass loss }) \\
{[\%]}\end{array}$ & $\begin{array}{l}\text { Porosity } \\
(\text { EtOH) } \\
{[\%]}\end{array}$ & 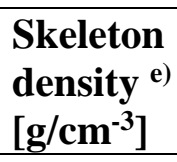 \\
\hline P0 & $0.2023 \pm 0.0022$ & $2.1 \pm 0.7$ & $80.4 \pm 0.3$ & $81.7 \pm 1.0$ & $1.08 \pm 0.1$ \\
\hline P10 & $0.2034 \pm 0.0018$ & $2.7 \pm 1.0$ & $79.2 \pm 0.4$ & $82.8 \pm 0.6$ & $1.08 \pm 0.1$ \\
\hline P20 & $0.2165 \pm 0.0038$ & $2.6 \pm 0.6$ & $79.1 \pm 0.6$ & $82.7 \pm 0.3$ & $1.09 \pm 0.1$ \\
\hline P30 & $0.2344 \pm 0.0083$ & $2.2 \pm 0.5$ & $79.4 \pm 0.1$ & $83.2 \pm 0.6$ & $1.10 \pm 0.1$ \\
\hline P40 & $0.2203 \pm 0.0042$ & $1.6 \pm 1.2$ & $78.9 \pm 0.7$ & $82.8 \pm 0.5$ & $1.10 \pm 0.1$ \\
\hline
\end{tabular}

a) retrieved from dividing the mass of the specimens by their volume; ${ }^{b}$ percentage of the specimens volume compared to the molds volume; ${ }^{c)}$ calculated from the mass loss during polymerization taking the volume change in consideration; ${ }^{\text {d) }}$ calculated from mass increase by immersion of EtOH taking the volume change into consideration; ${ }^{\text {e) }}$ calculated from mass and assuming $80 \%$ porosity
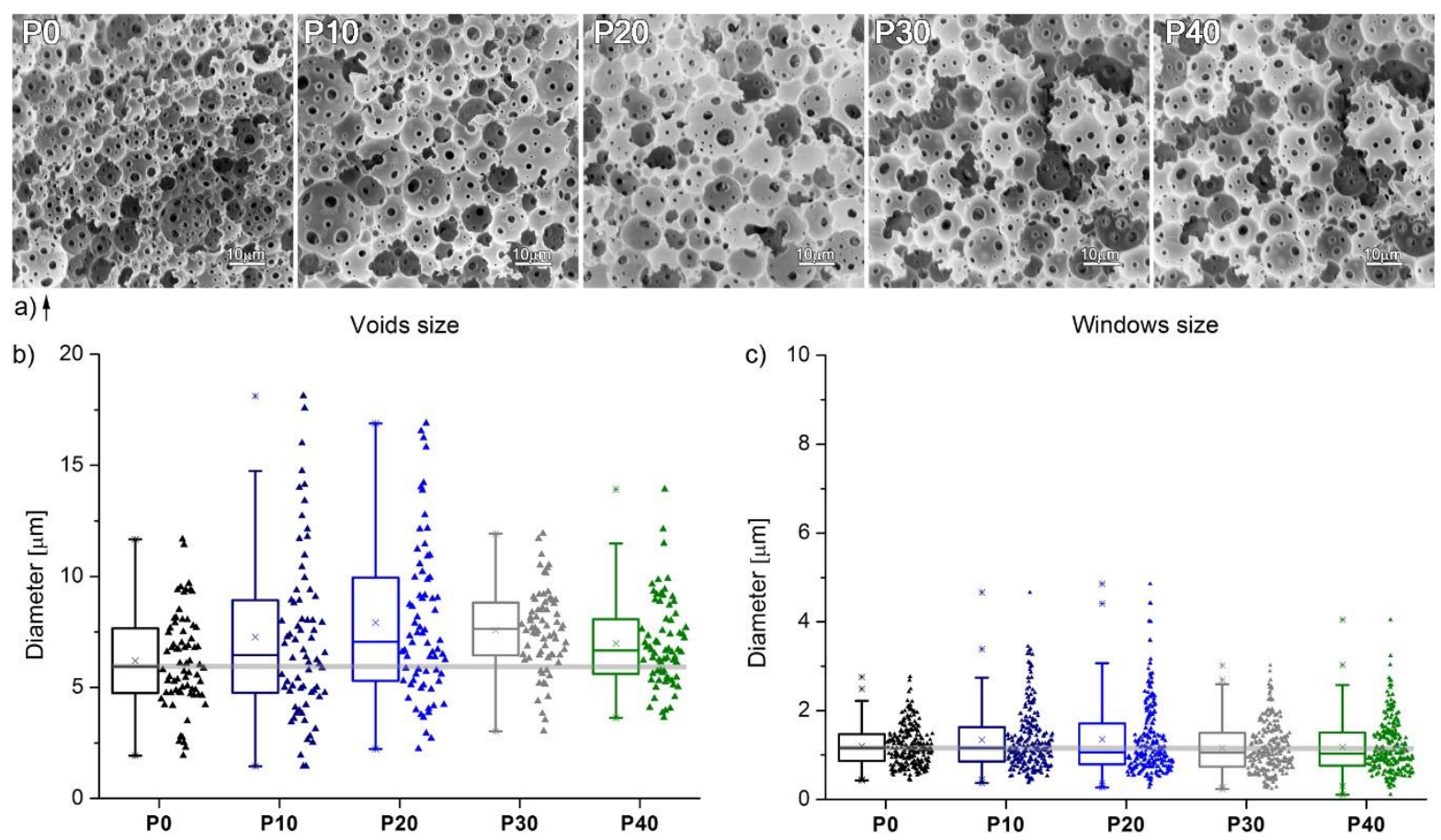

Figure 2. a) scanning electron micrographs of the (oxidized) foams P0-P40; box-whisker/bee swarm plot of b) void and c) window size distributions (median: horizontal line in the box; quartiles: upper and lower limit of the box, the boxes represent a restricted area where half of the overall values are included; whiskers: $1.5 \cdot \mathrm{IQR})$. The grey bars visualize the median voids and windows diameter for $\mathbf{P 0}$.

interconnected with windows. Voids and windows sizes were assessed from SEM measurements of broken samples and results are gathered in Figure 2. Compared to the 
reference sample P0 $(5.95 \pm 2.05 \mu \mathrm{m})$ all median voids diameter of $\mathbf{P 1 0}-\mathbf{P 4 0}$ are larger (in the range of $6.67 \pm 3.55-7.95 \pm 3.39$ ). The distribution of the diameters becomes distinctly larger when going from P0 to P10 and P20 and becomes even smaller in P30 and P40 (Figure 2b). Windows diameter and their distribution stay similar with values from $1.16 \pm 0.42$ to 1.36 $\pm 0.81 \mu \mathrm{m}$ over the whole series (Figure $2 \mathrm{c}$ ).

\subsection{Investigation of the chemical composition}

Infrared spectroscopy revealed the presence of CLC and the surfactant in the polymeric skeleton of the foam (Figure 3 ). The $\mathrm{C}=\mathrm{O}$ stretching vibration of the ester's carbonyl group was detected at $1727 \mathrm{~cm}^{-1}$ and is gradually decreasing with decreasing CLC loading from P40 to P10. A very small absorption at this wavelength was also detected in $\mathbf{P 0}$ and is ascribed to minor oxidation of pDCPD. ${ }^{[17]}$ The C-O stretching vibration of the C-O bonds in CLC was observed at $1169 \mathrm{~cm}^{-1}$ just next to the $\mathrm{C}-\mathrm{O}$ stretching vibration of the ether groups in Pluronic L121 $\left(1105 \mathrm{~cm}^{-1}\right)$. Accordingly, IR revealed that the surfactant was not completely removed from the samples during the extraction step. Comparing IR spectra of a $\mathbf{P 4 0}$ sample before and after extraction makes clear that the surfactant content is reduced by this step. Although all samples were treated in the same way, the amount of Pluronic L121 is different in all samples being highest in P20. It is plausible to assume that increasing the CLC share is facilitating the accommodation of Pluronic L121 in the nonpolar phase. However, the decrease of Pluronic L121 content at higher CLC loadings cannot be explained. The polymer backbone is characterized by $\mathrm{C}-\mathrm{H}$ bending vibrations of double bonds from trans-connected repeating units $\left(973 \mathrm{~cm}^{-1}\right)$ and $c i s$-connected repeating units as well as from cyclic olefins (755, 732 and 706 $\mathrm{cm}^{-1}$ ). Vibrations from either ring-opened or not ring-opened CLC and pDCPD superimpose in a way that a quantification of crosslinking from these data is not possible. 

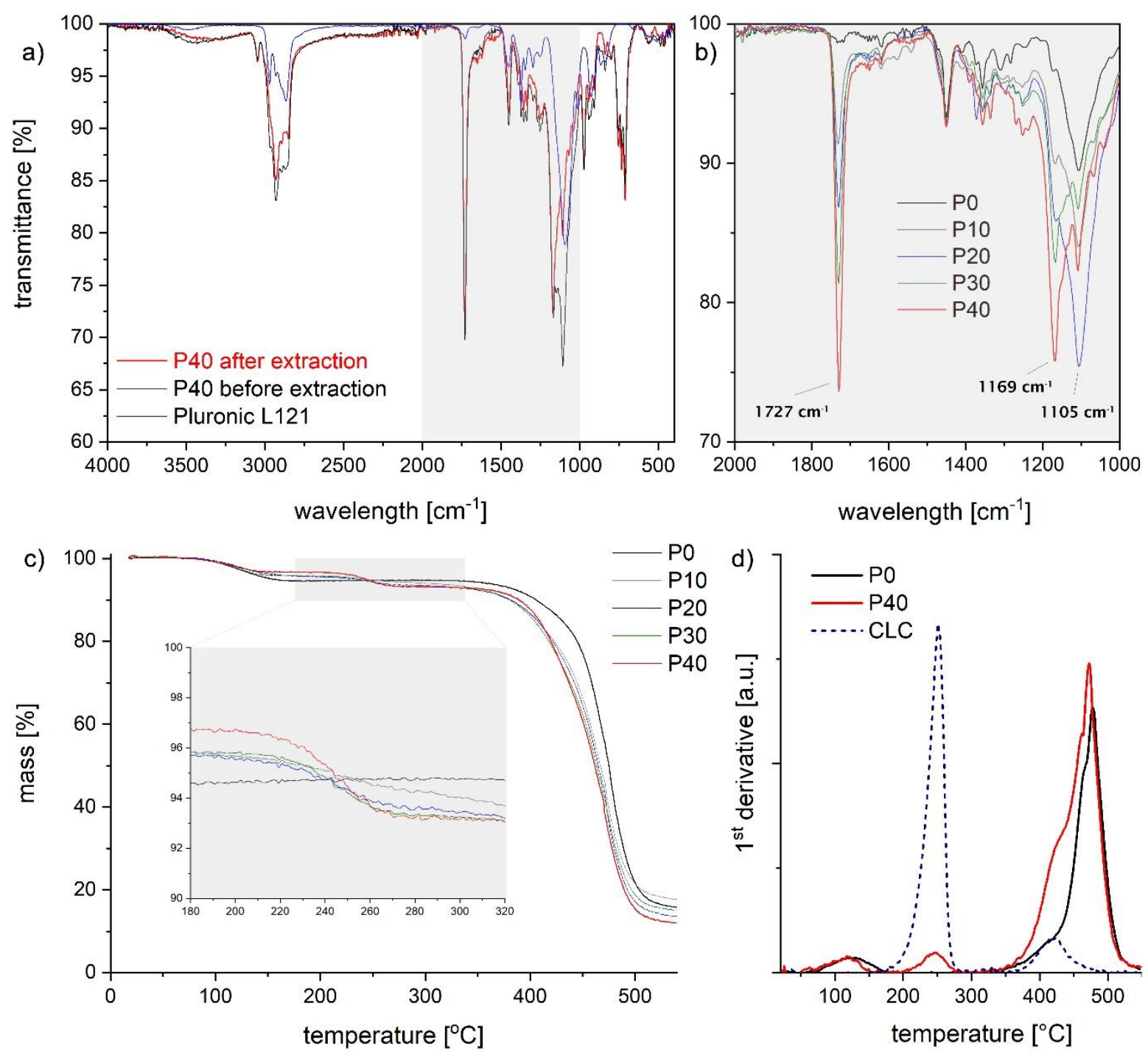

Figure 3. a) IR spectra of $\mathbf{P 4 0}$ after and before extraction and of Pluronics L121; b) detail of the IR spectra of P0-P40; c) TGA curves of P0-P40 showing in the inset a magnification of the mass loss due to the retro Diels-Alder reaction of free norbornene groups from CLC; d) first derivative of the TGA results for P0, P40 and the monomeric CLC.

Thermal gravimetric analysis (TGA) allows quantifying residual (not polymerized) norbornene ester moieties of CLC. A mass loss characterized by an inflection point at around $240^{\circ} \mathrm{C}$ is attributed to the retro-Diels Alder reaction of norbornene ester moieties of CLC. As evident from TGA of the CLC monomer at that temperature, cyclopentadiene and the corresponding acrylate is formed (Figure $3 \mathrm{~d}$ and Figure S5-S6). Both are volatile at $240^{\circ} \mathrm{C}$ and evaporate while a smaller share thermally polymerizes forming presumably a polyacrylate (which composes at slightly lower temperatures than then ROMP derived polymer). With this knowledge, the mass 
loss in P10-P40 can be attributed to the evaporation of cyclopentadiene and the ratio of polymerized to unpolymerized norbornene moieties of CLC can be quantified. Data reveal that approx. in half of the CLC molecules one norbornene is not ring-opened. Assuming that every doubly ring-opened CLC is a junction point, a maximal crosslinking degree originating from CLC can be calculated. Values amount to 1.7, 4.1, 7.6 and 10.8 mol\% junction points in P10, P20, P30 and P40, respectively. In other words, the average chain in between a junction point bears about 60 repeating units in $\mathbf{P 1 0}$ and about 10 in P40. The overall crosslinking degree, which should also take a ring opening of DCPD's cyclopentene ring into account, could not be assessed. Further information gained from TGA comprise a minor mass loss of $3 \pm 1.5 \mathrm{w} \%$ starting at $85^{\circ} \mathrm{C}$. The inclination point was found at $123 \pm 5^{\circ} \mathrm{C}$. This mass loss is most probably due to evaporation of toluene, which cannot be fully removed upon the drying procedure employed. Some evidence for this hypothesis comes from TGA investigations of bulk samples, i.e. non porous variants of the porous samples discussed here, which do not exhibit similar mass losses (Figure S6). Beside the discussed retro-Diels Alder reaction, the thermal stabilities of P10-P40 are slightly lower than that of P0, which shows thermal degradation at temperatures above $350^{\circ} \mathrm{C}$.

\subsection{Selling and Deswelling}

The uptake capability of the foams P0-P40 of different solvents was assessed by determining the weight and volume of disc-shaped specimens in wet state. Water, ethanol, $n$-pentanol, acetone and toluene were tested. Results are gathered in Figure 4a and b. Polar protic and polar aprotic solvents are accommodated mainly in the voids because no or low swelling of the specimens was detected. The water uptake $(334 \mathrm{w} \%)$ is somewhat lower than the calculated value (387-399 w\%) taking the range of the determined porosity of 79-83\% into account. This observation might be best explained by the high surface tension of water, which does not permis 
water to enter smaller structures. On the other hand, intrusion of water is nevertheless possible pointing to the presence of a polar surface of the foams likely formed by residual surfactant residing at the polymer's surface. Data retrieved for ethanol were used to determine the porosity (Table 1) albeit swelling was a little bit more pronounced than in the case of water. Less polar $n$-pentanol and acetone cause slightly more swelling than ethanol. The swelling increases when going from P0-P40 probably because of the increasing amount of CLC
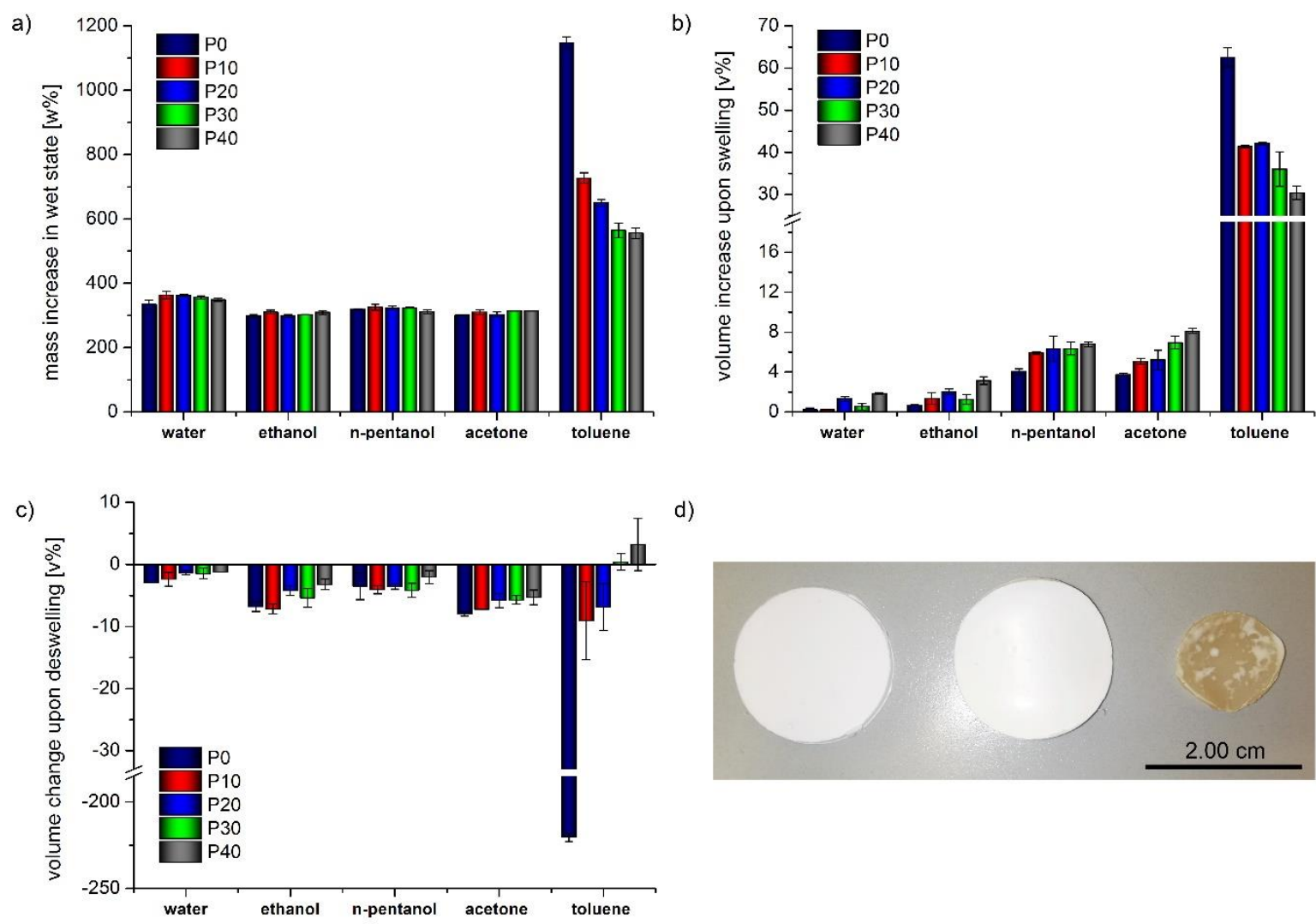

d)

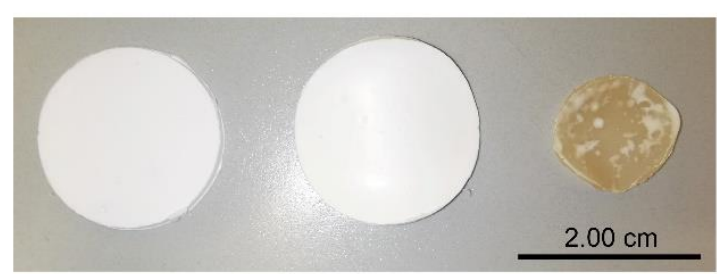

Figure 4. a) mass increase upon immersion of solvents; b) swelling of the samples; volume change upon drying the swollen samples expressed as $\mathrm{v} \%$ in respect to the volume before swelling; d) photographs of a typical specimen in wet state directly after preparation (left), specimen of P30 in dry state after swelling in toluene (middle), specimen of P0 in dry state after swelling in toluene (right).

that provides additional crosslinking but also polar aprotic ester groups to the polymer scaffold. In contrast, solvent uptake and swelling with nonpolar toluene is high. Both parameters are decreasing with increasing CLC content. While P0 takes up $1148 \pm 19$ w\% toluene upon swelling by $63 \pm 2.3 \mathrm{v} \%$, P40 accommodates only $555 \pm 16 \mathrm{w} \%$ toluene upon swelling by 
$30 \pm 1.5 \mathrm{v} \%$. A combination of the higher crosslinking degree and a more polar polymeric phase is held responsible for the observed trend (Figure $4 a$ and b). Successively the swollen specimens were dried under ambient conditions until constant mass was observed. In all cases complete drying was found, i.e. within experimental error masses after drying were the same as those measured before swelling. However, samples shrunk to a different degree when compared to the dimensions before immersion into the solvents (Figure 4c). The shrinkage is rather insignificant when water has been accommodated and amounts to 1-3 v\%. Less polar solvents lead to higher volume shrinkage of in the range of 2-8 v\% (ethanol, $n$-propanol and acetone) but in all cases the original disc shape of the specimens was conserved. In case of the least polar solvent toluene and P0 the pronounced shrinkage of $221 \mathrm{v} \%$ was observed, i.e. the original shape of specimens was lost (Figure 4d). In contrast, CLC-bearing foam specimens P10-P40 recovered their original shape showing low $(\mathbf{P 1 0}, \approx 9 \mathrm{v} \% ; \mathbf{P 2 0} \approx 7 \mathrm{v} \%)$ or no $(\mathbf{P 3 0}, \approx 0 \mathrm{v} \%)$ volume shrinkage. P40 even expanded by on average 3 v\%. In all solvent cases the lowest shrinkage upon drying was observed for $\mathbf{P 4 0}$ featuring the highest CLC loading. Specimens of P10-P40 were again tested for their porosity and their morphology and no significant difference in respect to the values from before swelling could be noted (Figure S7 and S8). This behavior

can be regarded as a solvent programmed shape memory effect. ${ }^{[42]}$ The specimens are programmed during molding, exhibit an omnidirectional swelling in toluene and recover their original shape upon drying or changing the solvent for acetone. The (higher) crosslinking in P10-P40 is supposed to be responsible for the effect.

\subsection{Mechanical Properties}

Finally, the mechanical properties of the foams were assessed by tensile testing and results are gathered in Figure 5 (and Table S1). In essence, it became clear that the ductility is decreasing while the stiffness of the samples is increasing with increasing CLC content (Figure 5a). The 
Young's modulus (reflecting the stiffness) of the polymer foams is steadily increasing from $103 \pm 3 \mathrm{MPa}$ for $\mathbf{P 0}$ to $117 \pm 3 \mathrm{MPa}$ in $\mathbf{P 4 0}$ (Figure $5 \mathrm{~b}$ ).
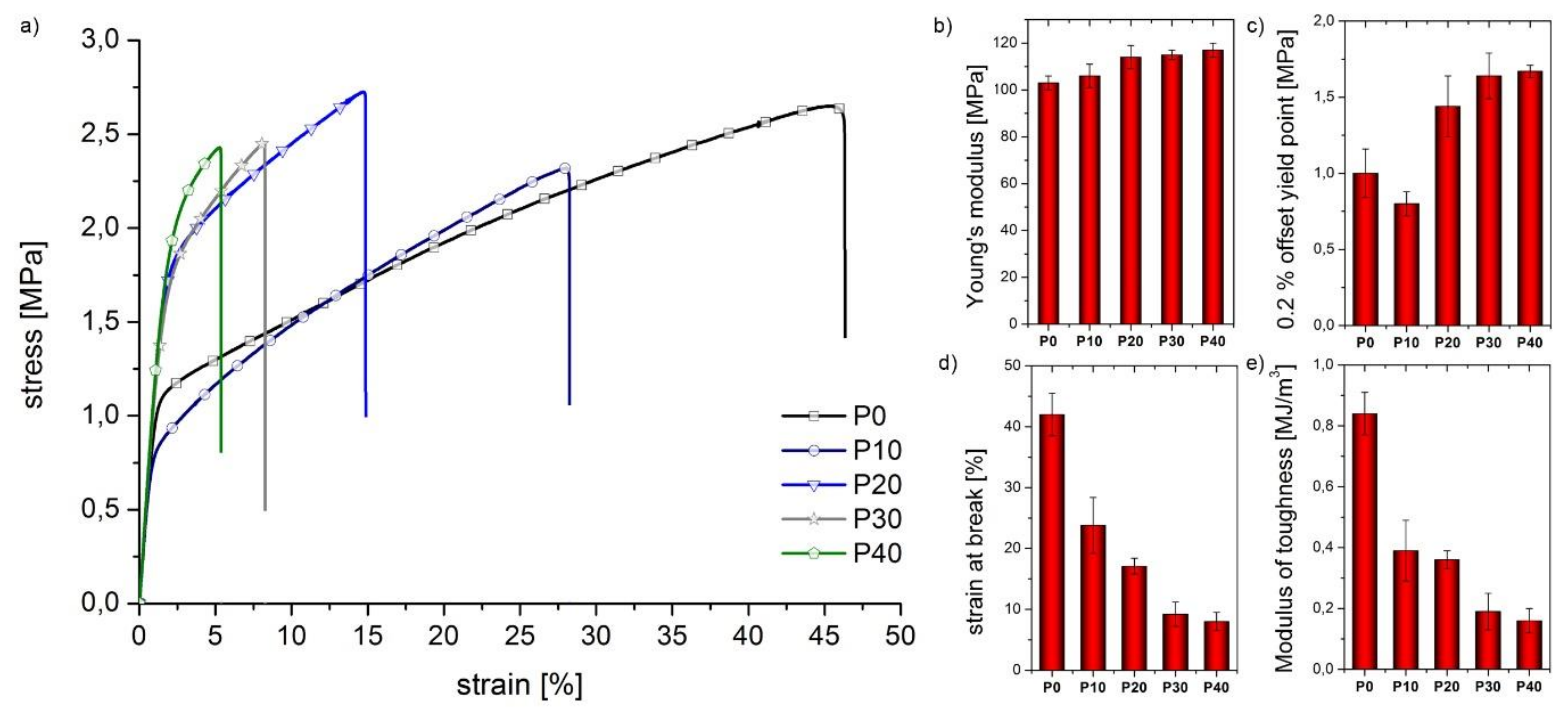

Figure 5. a) stress strain curves for foams P0-P40; diagrams of the b) Young's modulus; c) $0.2 \%$ offset yield strengths; d) strain at break and e) modulus of toughness of P0-P40.

These values are higher than the Young's modulus of carefully extracted pDCPD foams prepared with $7 \mathrm{v} \%$ Pluronic L121 ${ }^{[19]}$ and approach those of pDCPD foams prepared with 3 v\% Pluronic L121. ${ }^{[21]}$ The yield strength (determined at $0.2 \%$ offset) is dropping when going from $\mathbf{P 0}(1.00 \pm 0.16 \mathrm{MPa})$ to $\mathbf{P 1 0}(0.80 \pm 0.08 \mathrm{MPa})$ but is then again increasing with increasing CLC amount reaching 1.67 $\pm 0.04 \mathrm{MPa}$ in $\mathbf{P 4 0}$ (Figure 5c). A similar picture is retrieved for the ultimate tensile strength, which is highest in P20 $(2.72 \pm 0.24 \mathrm{MPa})$ and lowest in P10 $(2.18 \pm 0.21 \mathrm{MPa})($ Table S1). Both parameters are for all samples lower than those published for the two reference foams discussed above. ${ }^{[19,21]}$ In contrast, a comparatively high strain at break of $42.0 \pm 3.5 \%$ could be observed for P0. This high strain at break together with the appealing strength of $\mathbf{P 0}$ foams results in a high modulus of toughness of $0.84 \pm 0.07 \mathrm{MJ} \mathrm{m}^{-3}$. Actually, to the best of our knowledge, this is the highest modulus of toughness ever reported for a HIPE templated polymer foam of $80 \%$ porosity. Strain at break and the modulus of toughness are then decreasing with increasing CLC amount (Figure $5 \mathrm{~d}$ and e). It has been 
shown that feature sizes (in particular voids sizes) of the foam and the amount of surfactant remaining in the polymer skeleton are decisive factors for mechanical properties of HIPE templated foams. ${ }^{[21]}$ Herein, above that, the crosslinking density of the polymer skeleton is influenced by the various amounts of CLC in the formulation. The mechanical properties of P0 are different in respect to similar materials published in literature and that can be ascribed above all to the higher Pluronic L121 share remaining in the polymer skeleton. The surfactant is acting as a plasticizer causing a lowering of the offset yield strength and the ultimate strength and an increase of the strain at break. Switching to the formulations containing $\mathbf{C L C}$ three aspects have to be considered. First and most important, the CLC causes additional crosslinking, which results in decreasing ductility and increasing stiffness and strengths with increasing CLC loading. Second, the presence of low amounts of CLC allows more Pluronic L121 to be accommodated within the polymer phase, explaining the low offset yield strength and the relatively high strain at break in P10. Third, higher CLC loadings (i.e. denser polymer networks) more and more override the plasticizing effect of the surfactant leading to much stiffer and more brittle materials. These hypotheses are supported by the swelling/deswelling experiments in particular with toluene. As expected, increasing crosslinking in the series P0$\mathbf{P 4 0}$ is causing a decrease in toluene uptake and in swelling and is allowing for recovery of the original shape upon drying. Further support comes from the infrared measurements of the samples after extraction and drying. From there it is evident, that the surfactant amount is increasing from P0 to P20 and is then again decreasing from P20 to P30 and P40.

\section{Conclusions}

In summary, a series of polyHIPEs of $80 \%$ porosity using DCPD and a crosslinking comonomer CLC as the monomers were prepared and their properties in regard of varying CLC contents were studied. The maximum CLC loading amounted to $40 \mathrm{w} \%$ in respect to the overall monomer mass. High internal phase emulsions with higher CLC contents were not 
stable under the curing conditions. While porosity as well as voids and window sizes hardly change with increasing CLC amounts, the crosslinking degree produced from CLC is in increasing from 1.7 to $5.8 \%$ when going from P10 to P40. This crosslinking degree is distinctly influencing the mechanical properties of the foams, which become stiffer and less ductile upon increasing the CLC amount, i.e. upon enhancing the crosslinking degree. The CLC crosslinked foams considerably swell in toluene and recover their original shape and porosity upon drying. This feature distinguishes them from polyHIPEs made from dicyclopentadiene only as drying of toluene swollen $\mathbf{P 0}$ comes with severe shrinkage and a loss of porosity. Accordingly, the herewith introduced DCPD/CLC polyHIPEs are suitable for applications which demand repeated swelling and drying of the foams. Furthermore, they are perfectly set-up for postpolymerization functionalization reactions, which should be carried out in nonpolar solvents. In addition, the free norbornene groups introduced into the foams impart the porous materials an enhanced reactivity for post-polymerization functionalization reactions.

\section{Experimental Section}

\subsection{Syntheses}

4.1.1. Preparation of butane-1,4-diyl-bis(bicyclo[2.2.1]hept-5-ene-2-carboxylate) (CLC)

Freshly distilled cyclopentadiene $(8.06 \mathrm{~g}, 0.122 \mathrm{~mol}, 2.2 \mathrm{eq})$ was added dropwise to butane1,4-diyl diacrylate $(10.90 \mathrm{~g}, 0.055 \mathrm{~mol}, 1.0 \mathrm{eq})$ under stirring at a temperature of $4^{\circ} \mathrm{C}$. The temperature of the reaction mixture was held below $25{ }^{\circ} \mathrm{C}$ (cooling was necessary within the first $2 \mathrm{~h}$ ).After $6 \mathrm{~h}$ the reaction progress was monitored via ${ }^{1} \mathrm{H}-\mathrm{NMR}$ spectroscopy and according amounts of cyclopentadiene ( $1.6 \mathrm{~g})$ were slowly added to the reaction mixture, which was then heated to $40^{\circ} \mathrm{C}$ under constant agitation and kept there for $6 \mathrm{~h}$. As the sole by-product 
DCPD formed which was removed via flash column chromatography using cyclohexane as eluent. The desired product was eluted with Cy/EtOAc (3:1 (v:v)) in pure form. The solvents were distilled off and the residue was dried in vacuum. Yield: $16.72 \mathrm{~g}(92 \%)$, colorless liquid, exhibiting an endo:exo ratio of 4:1. $\mathrm{R}_{f}=0.64\left(\mathrm{Cy} / \mathrm{EtOAc}, 3: 1(\mathrm{v}: \mathrm{v}) ;{ }^{1} \mathrm{H}-\mathrm{NMR}\left(300 \mathrm{~Hz}, \mathrm{CDCl}_{3}\right)\right.$ : $\delta$ = $6.19\left(\mathrm{~m}, 2 \mathrm{H}, \mathrm{HC}=\mathrm{CH}_{\text {endo }}\right), 6,12\left(\mathrm{~m}, 1 \mathrm{H}, \mathrm{HC}=\mathrm{CH}_{\text {exo }}\right), 5.92\left(\mathrm{~m}, 2 \mathrm{H}, \mathrm{HC}=\mathrm{CH}_{\text {endo }}\right), 4.11(\mathrm{~m}, 2 \mathrm{H}$, $\mathrm{CH}_{2} \mathrm{CH}_{2} \mathrm{O}_{\text {exo }}$ ), 4.05 (s, 4H, $8 \mathrm{CH}_{2} \mathrm{O}_{\text {endo }}$ ), 3.20 (s, 2H, nb1 endo), 3,03 (s, 0.5H, nb1 $1_{\text {exo }}$ ), 2.97-2.91 (m, 4.5H, nb2 $\left.2_{\text {endo, }} \mathrm{nb} 1_{\text {endo }}, \mathrm{nb} 4_{\mathrm{exo}}\right), 2.21,1.93-1,86\left(\mathrm{~m}, 3 \mathrm{H}, \mathrm{nb} 3_{\text {endo }}, \mathrm{nb} 2_{\mathrm{exo}}, \mathrm{nb} 3_{\mathrm{exo}}\right), 1.68$ (m, 5H, $\mathrm{CH}_{2} \mathrm{CH}_{2} \mathrm{O}_{\text {endo/exo }}$ ), 1.42-1.28 (m, 7.5H, nb3 endo, nb7 endo, nb3 exo, nb7 exo). ${ }^{13} \mathrm{C}-\mathrm{NMR}\left(75 \mathrm{~Hz}, \mathrm{CDCl}_{3}\right)$ : $\delta=176.1\left(2 \mathrm{C}_{\text {exo }}, C \mathrm{OOCH}_{2}\right), 174.9\left(2 \mathrm{C}_{\text {endo }}, C \mathrm{OOCH}_{2}\right), 138.0\left(2 \mathrm{C}_{\text {exo }}, \mathrm{HC}=\mathrm{CH}\right), 137.9\left(2 \mathrm{C}_{\text {endo }}\right.$, $\mathrm{HC}=\mathrm{CH}), 135.7\left(2 \mathrm{C}_{\text {exo }}, \mathrm{HC}=\mathrm{CH}\right), 132.4\left(2 \mathrm{C}_{\text {endo }}, \mathrm{HC}=\mathrm{CH}\right), 63.9\left(2 \mathrm{C}, \mathrm{CH}_{2} \mathrm{CH}_{2} \mathrm{O}\right), 63.8(2 \mathrm{C}$, $\left.\mathrm{CH}_{2} \mathrm{CH}_{2} \mathrm{O}\right), 49.6,46,6,46,3,45.7,43.3,43.1,42.5,41.6,30.3,29.2$, 25.4. Elem. Anal. calcd: C, 72.70; H, 7.93; O, 19.37; found: C, 72.60; H, 8.06; O, 19.42 .

\subsubsection{Preparation of the foams $\mathbf{P 0}-\mathbf{P} 40$}

In a $250 \mathrm{~mL}$ three-necked round bottom flask a total amount of $10 \mathrm{~g}$ of monomers (according to Table 2), $0.7 \mathrm{~g}$ of the surfactant Pluronic L-121 (7 wt\% in respect to the total mass of monomers) and toluene $(100 \mu \mathrm{L})$ were added. The reaction mixture was stirred with a mechanical stirrer at $400 \mathrm{rpm}$ while $40 \mathrm{~mL}$ of distilled water was added dropwise over a period of $15 \mathrm{~min}$. Stirring of the formed emulsion was carried on for $1 \mathrm{~h}$. Then the initiator M2 (molar ratio of 1:15000 in respect to both monomers) dissolved in $200 \mu \mathrm{L}$ of toluene was added to the emulsion under agitation. The resulting pinky mixture was poured into molds, which were closed and cured at $80{ }^{\circ} \mathrm{C}$ for $3 \mathrm{~h}$ in an oven operated under air. The demolded specimens were submersed in acetone for $15 \mathrm{~min}$ and subsequently dried under ambient conditions until constant weight was reached. 
Table 2. DCPD, CLC and initiator amounts used

\begin{tabular}{lccc}
\hline Sample & $\begin{array}{l}\text { DCPD } \\
\text { [g] }([\mathbf{m m o l}])\end{array}$ & $\begin{array}{l}\text { CLC } \\
\text { [g] }([\mathbf{m m o l}])\end{array}$ & $\begin{array}{l}\text { Initiator M2 } \\
\text { [g] }([\mathbf{m m o l}])\end{array}$ \\
\hline P0 & $10.0(75.6)$ & - & $4.8(0.0050)$ \\
P10 & $9.0(68.1)$ & $1.0(3.0)$ & $4.5(0.0047)$ \\
P20 & $8.0(60.5)$ & $2.0(6.1)$ & $4.2(0.0044)$ \\
P30 & $7.0(52.9)$ & $3.0(9.1)$ & $3.9(0.0041)$ \\
P40 & $6.0(45.4)$ & $4.0(12.1)$ & $3.6(0.0038)$
\end{tabular}

\subsection{Characterization}

4.2.1. Swelling/deswelling procedure

The specimens for every copolymer composition were prepared according to 4.2.1. The weight and dimensions of dry samples were recorded. Five solvents exhibiting different polarities at room temperature, water (dielectric constant $\varepsilon=80)$, ethanol $(\varepsilon=25)$, $n$-pentanol $(\varepsilon=15)$, acetone $(\varepsilon=21)$ and toluene $(\varepsilon=2.4)$ were used for swelling/deswelling experiments. The specimens were immersed into the respective solvent for $24 \mathrm{~h}$ and following, the dimensions and weight of wet samples were determined. By calculating the volume for the dry and wet samples and setting those values in relation the swelling degree was retrieved. The solvent uptake was determined by measuring out the mass of the wet sample and was used for calculating the solvent uptake in w\%. Afterwards, the specimens were dried under ambient conditions until constant mass was obtained. The dimensions of the dry samples were measured and set into relation with the volume before the swelling procedure. For every formulation P0$\mathbf{P 4 0}$ at least five specimens were investigated.

\section{Supporting Information}

Supporting Information is available as separate file

Acknowledgements: EV thanks the Onassis Foundation for a stipend. The lead-project LP-03 of Graz University of Technology is acknowledged. We thank C. Wappl for elaborating the synthesis of CLC, R. Saf for SEM measurements and Umicore for providing initiator M2. 


\section{References}

[1] T. Zhang, R. A. Sanguramath, S. Israel, M. S. Silverstein, Macromolecules 2019, 52, 5445.

[2] M. S. Silverstein, Polymer 2017, 126, 261.

[3] K. M. L. Taylor-Pashow, Solvent Extr. Ion Exch. 2019, 37, 1.

[4] J. Zhu, L. Wu, Z. Bu, S. Jie, B.-G. Li, Ind. Eng. Chem. Res. 2019, 58, 4257.

[5] R. Zowada, R. Foudazi, ACS Appl. Polym. Mater. 2019, 1, 1006.

[6] S. Kovačič, N. Drašinac, A. Pintar, E. Žagar, Langmuir 2018, 34, 10353.

[7] Z. Wu, W. Hu, T. Huang, P. Lan, K. Tian, F. Xie, L. Li, J. Mater. Chem. C. 2018, 6, 8839.

[8] G. Tripodo, G. Marrubini, M. Corti, G. Brusotti, C. Milanese, M. Sorrenti, L. Catenacci, G. Massolini, E. Calleri, Polym. Chem. 2018, 9, 87.

[9] M. Corti, E. Calleri, S. Perteghella, A. Ferrara, R. Tamma, C. Milanese, D. Mandracchia, D. Brusotti, M. L. Torre, D. Ribatti, F. Auricchio, G. Massolini, G. Tripodo, Mater. Sci. Eng. C 2019, in press. DOI: 10.1016/j.msec.2019.110060

[10] M. Whitely, G. Rodriguez-Rivera, C. Waldron, S. Mohiuddin, S. Cereceres, N. Sears, N. Ray, E. Cosgriff-Hernandez, Acta Biomater. 2019, 93, 169.

[11] L. Weinstock, R. A. Sanguramath, M. S. Silverstein, Polym. Chem. 2019, 10, 1498.

[12] L. Yang, Y. Liu, C. D. M. Filipe, D. Ljubic, Y. Luo, H. Zhu, J. Yan, S. Zhu, ACS Appl. Mater. Interfaces 2019, 11, 4318.

[13] F. Du, L. Sun, W. Tan, Z. Wie, H. Nie, Z. Huang, G. Ruan, J. Li, Anal. Bioanal. Chem. 2019, 411, 2239.

[14] E. Vakalopoulou, S. M. Borisov, C. Slugove, ChemRxiv 2019. DOI: 10.26434/chemrxiv.9124730.v2

[15] H. Deleuze, R. Faivre, V. Herroguez, Chem. Commun. 2002, 2822. 
[16] K. Benmachou, H. Deleuze, V. Herroguez, React. Funct. Polym. 2003, 55, 211.

[17] S. Kovacic, P. Krajnc, C. Slugovc, Chem. Commun. 2010, 46, 7504.

[18] S. Kovačič, K. Jeřabek, P. Krajnc, C. Slugovc, Polym. Chem. 2012, 3, 325.

[19] S. Kovačič, N. B. Matsko, K. Jeřabek, P. Krajnc, C. Slugovc, J. Mater. Chem. A 2013, 1, 487 .

[20] E. H. Mert, C. Slugovc, P. Krajnc, Express Polym. Lett. 2015, 9, 344.

[21] S. Kovačič, E. Žagar, C. Slugovc, Polymer 2019, 169, 58.

[22] N. Trupej, Z. Novak, Ž. Knez, C. Slugovc, S. Kovačič, J. CO2 Util. 2017, 21, 336.

[23] A.-C. Knall, S. Kovačič, M. Hollauf, D. P. Reishofer, R. Saf, C. Slugovc, Chem. Commun. 2013, 49, 7325.

[24] C. L. McGann, G. C. Daniels, S. L. Giles, R. B. Balow, J. L. Miranda - Zayas, J. G. Lundin, J. H. Wynne, Macromol. Rapid Commun. 2018, 39, 1800194.

[25] R. B. Balow, S. L. Giles, C. L. McGann, G. C. Daniels, J. G. Lundin, P. E. Pehrsson, J. H. Wynne, Ind. Eng. Chem. Res. 2018, 57, 8630.

[26] S. Kovačič, F. Preishuber-Pflügl, D. Pahovnik, E. Žagar, C. Slugovc, Chem. Commun. 2015, 51, 7225.

[27] S. Kovačič, H. Kren, P. Krajnc, S. Koller, C. Slugovc, Macromol. Rapid Commun. 2013, 34,581 .

[28] S. Kovačič, N. B. Matsko, G. Ferk, C. Slugovc, J. Mater. Chem. A. 2013, 1, 7971.

[29] S. Kovačič, A. Anžlovar, B. Erjavec, G. Kapun, N. B. Matsko, M. Zigon, E. Zagar, A. Pintar, C. Slugovc, ACS Appl. Mater. Interfaces 2014, 6, 19075.

[30] E. Yüce, E. H. Mert, P. Krajnc, F. N. Parın, N. San, D. Kaya, H. Yıldırım, Macromol. Mater. Eng. 2017, 302, 1700091.

[31] E. Yüce, P. Krajnc, H. H. Mert, E. H. Mert, Appl. Polym. Sci. 2019, 136, 46913. 
[32] S. Kovačič, M. Mazaj, M. Jeselnik, D. Pahovnik, E. Žagar, C. Slugovc, N. Zabukovec Logar, Macromol. Rapid Commun. 2015, 36, 1605.

[33] M. Mazaj, N. Zabukovec Logar, E. Žagar, S. Kovačič, J. Mater. Chem. A 2017, 5, 1967.

[34] M. Scharfegger, B. Fuchsbichler, S. Koller, C. Slugovc, S. Kovacic, (Varta Micro Innovation GmbH, Technische Universität Graz) WO2013178371A1, 2013.

[35] S. M. Andler, J. M. Goddard, J. Agric. Food Chem. 2018, 66, 3619.

[36] S. Kovačič, F. Preishuber-Pflügl, C. Slugovc, Macromol. Mater. Eng. 2014, 299, 843.

[37] A.-C. Knall, C. Slugovc, Chem. Soc. Rev. 2013, 42, 5131.

[38] A.-C. Knall, M. Hollauf, C. Slugovc. Tetrahedron Lett. 2014, 55, 4763.

[39] C. E. Hoyle, C. N. Bowman, Angew. Chem. Int. Ed. 2010, 49, 1540.

[40] C. N. Walker, J. M. Sarapas, V. Kung, A. L. Hall, G. N. Tew, ACS Macro Lett. 2014, 3, 453.

[41] A. Leitgeb, J. Wappel, C. A. Urbina-Blanco, S. Strasser, C. Wappl, C. S. J. Cazin, C. Slugovc, Monatsh. Chem. 2014, 145, 1513.

[42] D. Chen, X. Xia, T. W. Wong, H. Bai, M. Behl, Q. Zhao, A. Lendlein, T. Xie, Macromol. Rapid Commun. 2017, 38, 1600746. 